\title{
Does the pilocarpine phenylephrine provocative test help in the management of acute and subacute angle closure glaucoma?
}

\author{
P K Wishart
}

\begin{abstract}
The pilocarpine phenylephrine provocative test (PPPT) has been described as a highly sensitive method to identify eyes suffering from or at risk of angle closure glaucoma (ACG). ${ }^{1}$ This paper reports on average 10 years follow-up of the outcome of cases of primary acute ACG and subacute ACG in which the management was determined by the result of the PPPT - a positive result indicating the need for a peripheral iridectomy, a negative result conservative treatment. In spite of a negative PPPT the fellow eyes of cases of acute ACG treated conservatively had a high rate $(40 \%)$ of development of ACG. Similarly, in eyes with a history of subacute ACG with a negative PPPT, ACG developed at some stage during the follow-up in $60 \%$. Peripheral iridectomy alone resulted in normal intraocular pressure in $63 \%$ of eyes that had suffered an attack of acute ACG. In hypertensive eyes that presented with subacute ACG, however, only one eye became normotensive following peripheral iridectomy alone. In the subacute ACG group a positive PPPT was closely related to the presence of glaucomatous optic disc damage.

These results indicate that the PPPT lacks sensitivity in detecting eyes at risk of angle closure glaucoma, and a positive result is likely in eyes with damaged outflow that will not respond to peripheral iridectomy.
\end{abstract}

Angle closure glaucoma (ACG) is believed to occur when a relative degree of pupil block leads to iridocorneal contact and subsequent closure of the trabecular meshwork by the peripheral iris. Primary ACG occurs in eyes with shallow anterior chambers (AC) and narrow angles. ${ }^{2}$ Many more people have narrow angles and shallow ACs than develop ACG, and the risk of the individual is difficult to assess. ${ }^{3}$ The symptoms and signs of a case of acute ACG - pain and blurred vision, corneal oedema, very high intraocular pressure (IOP), and a mid-dilated fixed, oval pupil - are well recognised, and treatment is directed at the relief of pupil block. ${ }^{4}$ Patients with shallow ACs presenting with a history suggestive of an attack of subacute ACG may present more of a diagnostic challenge. The differential diagnosis includes all causes of ocular or periorbital pain, but in an eye with raised pressure and a narrow angle the clinician is likely to think first of subacute ACG. The desire for an objective test that will indicate the presence of angle closure or identify those eyes especially at risk has led to the development of provocative

tests. This paper examines the use of the pilocarpine phenylephine provocative test (PPPT) to identify those eyes at risk of ACG and those eyes in which raised IOP was due to an angle closing mechanism.

\section{Patients and methods}

Between 1974 and 1986 patients presenting with acute or subacute ACG were entered into a longitudinal study. In cases of acute ACG with a fixed, semidilated pupil, corneal oedema, ocular pain, and IOP greater than $45 \mathrm{mmHg}$ surgical peripheral iridectomy was performed once the IOP had been lowered by medical therapy. All eyes with signs and/or a history of symptoms suggestive of subacute ACG and the fellow eye of cases of acute ACG were treated by performing a PPPT soon after presentation. If negative, the test would be repeated at approximately three months after presentation and thereafter at yearly intervals. If at a later date the test became positive - and a rise of $8 \mathrm{mmHg}$ in the presence of a closed angle was taken as a positive result - a peripheral iridectomy would be performed. Miotics were not prescribed prophylactically for those eyes with a negative PPPT, but a raised IOP was treated with timolol and/or pilocarpine. Since 1987 all patients in this study was examined by Zeiss four-mirror indentation gonioscopy to identify peripheral anterior synechiae of the AC angle. Axial anterior chamber depth was measured with the Haag-Streit pachymeter, and a full slit-lamp examination of the anterior and posterior segments of both eyes was performed.

\section{Results}

One hundred and fifty-eight patients with acute or presumed subacute ACG were entered into the study, but 18 were either dead or lost to follow-up by December 1986 . In seven cases the ACG was secondary to an event such as central retinal vein occlusion or intumescent lens, and these patients have been excluded from this report. Two patients with a diagnosis of subacute ACG are excluded, as one patient's symptoms were later shown to be due to migraine, and in the other presentation was following a rise in pressure due to the instillation of mydriatics for a routine fundus examination.

One hundred and four patients presented with acute ACG. Thirty two patients (64 eyes) with at least two of the following symptoms or signs of subacute ACG - pain, blurring, spectral haloes, and raised IOP - were entered into the study and followed up. The demographic details of these patients are given in Fig 1 and 2 and the
Eye Hospital, Old Hall

P K Wishart

Correspondence to:

Mr P K Wishart, FRCSGlasg.

Accepted for publication

13 November 1990 


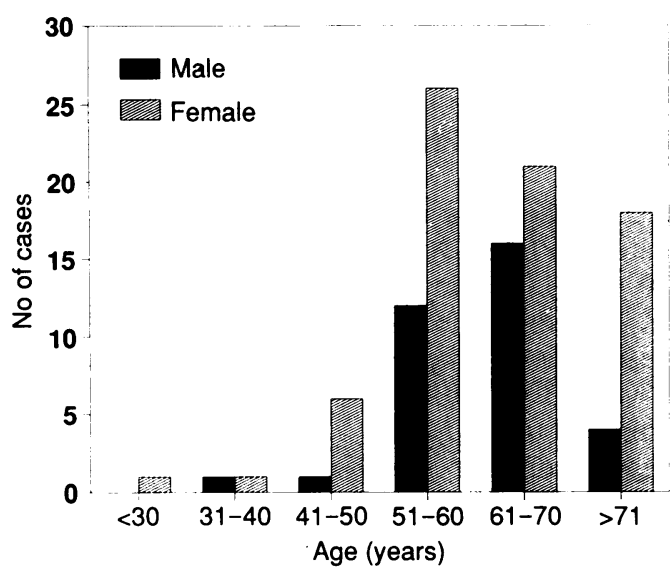

Figure 1 Patients with acute ACG: age in years.

distribution of axial anterior chamber depths in Fig 3 and 4.

In common with other studies acute ACG was seen more commonly in women than men (74 females to 30 males). An onset in the winter months (October to March) was more common in cases of acute ACG, with 75 in winter and 29 in summer. In the patients with subacute ACG the proportion of females to males was (20:12), with 14 winter onsets to 18 summer onsets. The average age was 61.7 (SD 8.7) years in acute ACG patients and 57.45 years (SD 8.94) in subacute ACG patients. In acute ACG eyes the mean anterior chamber depth was $1.72 \mathrm{~mm}$, (SD $0 \cdot 297$ ), in fellow eyes $1.765 \mathrm{~mm}(\mathrm{SD} 0.293)$, and in subacute ACG $1.91 \mathrm{~mm}$ (SD $0.23 \mathrm{~mm}$ ).

The mean follow-up of the patients in the acute ACG group was 9.95 years (SD 5.66), and in the subacute ACG group 10.7 years (SD 4.02).

By the end of the follow-up period, of the 104 eyes that had suffered acute ACG one eye had been enucleated because it was blind and painful. Twenty-six eyes had raised IOP requiring longterm hypotensive medication, and in a further 12 eyes the pressure was normal after successful filtering surgery. Peripheral iridectomy alone resulted in a normal IOP in $65(63 \%)$ of the eyes that had suffered an attack of acute ACG.

In the acute ACG patients, of the 104 fellow eyes 37 showed a positive PPPT. In 20 eyes this test was positive within 10 days of presentation, and in a further 13 eyes the test was positive within the first year following presentation. In the remaining four fellow eyes the PPPT

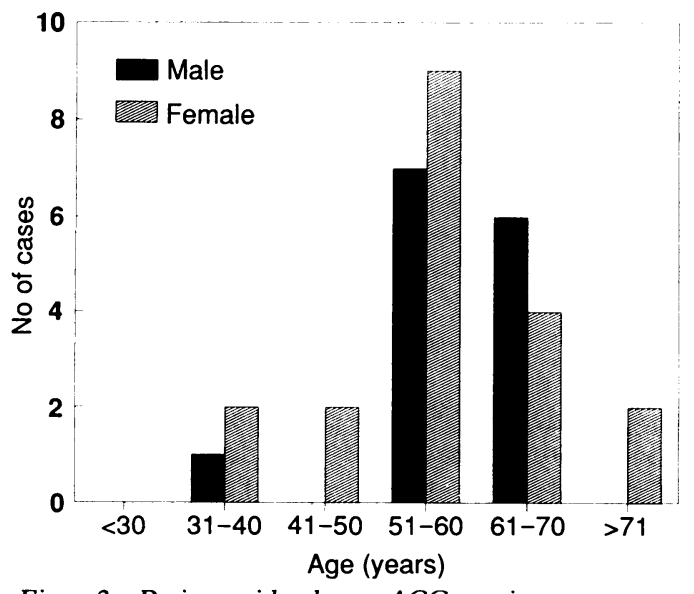

Figure 2 Patients with subacute ACG: age in years.

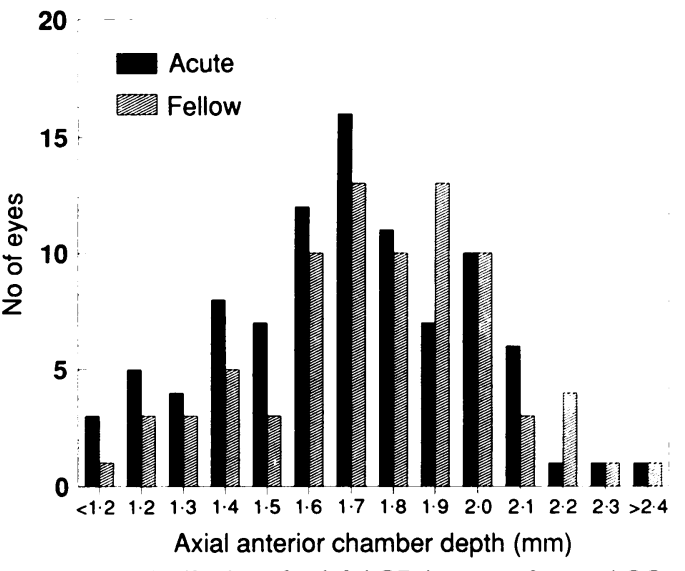

Figure 3 Distribution of axial ACD in cases of acute ACG.

became positive between one and 10 years after presentation. Of these 37 fellow eyes with a positive PPPT 34 underwent a prophylactic surgical peripheral iridectomy, one was treated by lens extraction, and two had prophylactic argon laser iridotomies performed. The 35 eyes that were treated surgically had a satisfactory outcome in that no eye subsequently developed angle closure glaucoma or required further surgery. Both eyes that underwent argon laser iridotomy developed raised IOP, and the laser iridotomies were judged to be non-patent. Because of the raised IOP and the laser-induced intraocular inflammation both these eyes underwent surgical trabeculectomy. Prior to their prophylactic surgical peripheral iridectomy nine of these fellow eyes had a mild increase of the IOP (five eyes being in the group that had a positive PPPT within 10 days of presentation, and four in the group whose PPPT became positive later). The IOP remained raised in all these nine eyes postoperatively and was treated with topical medication. In only one eye was glaucomatous damage to the optic disc observed by the end of the follow-up period. The other eyes were judged to be suffering from ocular hypertension, while their optic discs remained normal.

In four of the remaining 67 fellow eyes a PPPT induced an attack of acute angle closure glaucoma unresponsive to 24 hours of medical therapy and requiring surgery for control. Four cases

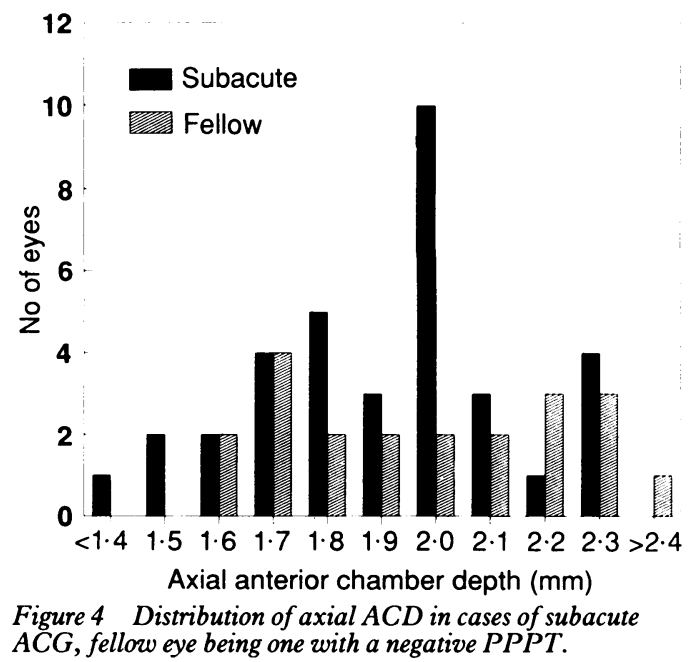


presented with bilateral acute ACG, and one developed acute ACG while waiting for PPPT of the fellow eye. One fellow eye with an anterior chamber depth of $2.7 \mathrm{~mm}$ was thought not to be at risk of ACG.

Fifty six at risk fellow eyes in the acute ACG group had consistently negative PPPTs. During two to 15 years (average 10 years) of follow-up despite a negative PPPT, 14 spontaneously developed an attack of acute ACG, and three eyes developed symptoms of subacute ACG. A further five eyes had persistently raised IOP, and Zeiss four-mirror indentation gonioscopy revealed extensive synechial closure of the superior anterior chamber angle. These eyes were asymptomatic and were therefore cases of creeping ACG as described by Lowe. ${ }^{5}$ Thus 22 of 56 $(40 \%)$ at risk fellow eyes with a negative PPPT developed closed-angle glaucoma.

In the subacute ACG patients 30 eyes had a positive PPPT, 27 of these within the first two months of presentation, and underwent peripheral iridectomy. Twenty of these eyes with positive PPPT had a raised IOP at the time of presentation, and 10 were normotensive. All 10 normotensive eyes had normal discs, but only seven of the hypertensive eyes had normal discs. The other 13 eyes in the hypertensive group had advanced glaucomatous optic disc damage.

In those 20 hypertensive eyes with a positive PPPT, peripheral iridectomy resulted in a normal IOP without further treatment only in one eye. In a further two eyes it improved control of the IOP. In the other 17 eyes the IOP was not lowered by the operation. Five eyes required trabeculectomy, and 11 had progressive, severe visual loss due to glaucomatous optic disc damage by the end of the follow-up period. Nine eyes developed cataracts.

One eye in the patients with suspected subacute ACG is excluded from the analysis because it had suffered previous angle recession, and therefore 33 eyes in the subacute ACG group had negative PPPTs, of which 18 were hypertensive at the time of presentation. Thirty two of these negative PPPT eyes had normal optic discs, while one showed advanced glaucomatous damage.

Of these 33 remaining eyes followed up with yearly negative PPPTs six spontantously developed an attack of acute ACG, and nine developed signs and symptoms of subacute ACG. Five eyes with persistently raised IOP were seen to have extensive closure of peripheral anterior synechial of the superior anterior chamber angle and were therefore judged to have developed creeping ACG. ${ }^{6}$

Four eyes in the negative PPPT group developed cataracts, but all these occurred in eyes that had suffered a spontaneous attack of acute ACG. Thus 20 of the $33(60 \%)$ eyes at risk with a negative PPPTs developed ACG.

\section{Discussion}

Observation of a large group of patients at risk of developing ACG was considered to be justified by means of the PPPT to identify the ones at risk and those eyes in which the raised IOP was due to an angle closing mechanism. ${ }^{16}$ Views differ on the reliability of provocative tests. Spaeth ${ }^{7}$ has criticised all pharmacological provocative tests as being unphysiological, especially the PPPT, which induces a situation never occurring in life - that of maximum sympathetic and parasympathetic forces operating simultaneously in the eye. He recommends, if any is to be used, the dark-room test, which has been unfavourably compared to the PPPT by Mapstone, ${ }^{8}$ who reports the PPPT as being $93 \%$ sensitive for detection and prediction of ACG.'

Although many ophthalmologists consider that the risk of ACG in a fellow eye is so great that a prophylactic or laser iridotomy should always be performed, ${ }^{910}$ others have disagreed, preferring reliance on provocative tests to determine which fellow eye should undergo surgical peripheral iridectomy. ${ }^{1112}$ For patients with acute ACG most surgeons nowadays perform a prophylactic laser iridotomy on fellow eyes owing to the relative ease and safety of this procedure. However, both argon and YAG laser iridotomy may fail to relieve pupil block. ${ }^{13}$ Usually this occurs as a result of closure of the iridotomy by the reparative process of pigment epithelial proliferation. ${ }^{13} \mathrm{~A}$ very small iridotomy in a thick iris may also be unable to permit sufficient flow of aqueous from the posterior to the anterior chamber to break the pupil block. ${ }^{13}$ Some authors have recommended that provocative testing should be performed following laser iridotomy, ${ }^{14}{ }^{15}$ and a recent paper has suggested the use of the PPPT to ensure adequacy of the iridotomy. ${ }^{16}$

The PPPT has been reported as a highly sensitive method of detecting the presence of ACG and predicting whether an eye is likely to suffer from it. ${ }^{1}$ In a paper which included cases of acute ACG, subacute ACG, and chronic ACG Mapstone reported that the risk of ACG occurring more than 5 years after an attack of ACG was nil. ${ }^{1}$ Further follow-up of these cases with primary symptomatic ACG showed that spontaneous acute ACG can occur more than five years after the initial attack (three eyes), and $40 \%$ of fellow eyes developed ACG not detectable by the PPPT. The long-term sensitivity of the PPPT must therefore be only $61 \%$, a relatively low figure considering that fellow eyes are known to be at high risk of angle closure glaucoma. ${ }^{17} 18$

In those eyes with a history of subacute ACG a negative PPPT was associated with the later development of ACG in $60 \%$. It is important to note also that only one eye with a damaged optic disc had a negative PPPT, while the other 14 $(93 \%)$ eyes with glaucomatous discs had positive PPPTs. If these eyes are analysed with respect to raised IOP, of 21 eyes with raised IOP and a positive PPPT 14 (66\%) had severe disc damage, while of 18 hypertensive eyes with a negative PPPT only one eye (6\%) showed disc damage. Thus an eye with raised IOP and a damaged optic disc is 10 times more likely to have a positive than a negative PPPT.

Peripheral iridectomy alone did not result in a normal IOP in any hypertensive eye with optic disc damage, yet $93 \%$ of such eyes had a positive PPPT. Clearly, if a PPPT in an eye with disc damage is positive and a peripheral iridectomy is performed, the IOP is unlikely to be lowered. There seems little point, therefore, in performing 
the PPPT in such eyes. From the findings in this study a positive result does not necessarily mean that an angle closing mechanism has been identified; it may simply occur because an already damaged outflow mechanism is unable to cope with pigment release, relative degree of induced pupil block, or the shallowing of the anterior chamber - all of which occur as a result of the PPPT ${ }^{19}{ }^{20}$ The PPPT therefore does not specifically identify that an angle closing mechanism is responsible for the raised IOP.

In contrast, peripheral iridectomy alone resulted in a normal IOP in $63 \%$ of cases of acute ACG in this study, which is similar to the $72 \%$ success rate reported by Playfair and Watson ${ }^{9}$ and the $69 \%$ success rate reported David $e t a l^{21}$ for acute ACG.

In this study prophylactic surgical peripheral iridectomy performed on those fellow eyes that had a positive PPPT was successful in preventing ACG. In the two fellow eyes where an attempt to create a prophylactic argon laser iridotomy failed, subsequent intraocular inflammation and raised IOP necessitated filtering surgery. However, at that time argon laser iridotomy was in its infancy, and the poor results represent failure of technique. With more modern techniques ${ }^{22}$ and newer lasers, ${ }^{23}{ }^{24}$ laser iridotomy is a widely accepted and very successful alternative to surgical peripheral iridectomy.

Finally, the PPPT itself has risks. Following a PPPT four fellow eyes of the acute ACG group and three from the subacute ACG developed an acute attack of ACG, which in $50 \%$ of cases required surgery to break the attack.

\section{CONCLUSIONS}

In any eye with primary ACG, a prophylactic surgical iridectomy or laser iridotomy should be performed on the fellow eye to prevent the development of ACG. The PPPT is neither a sensitive nor a specific test and is not helpful in the diagnosis or management of ACG.
I thank Mrs C Owen for technical and administrative help.

1 Mapstone R. The fellow eye. Br $\mathcal{F}$ Ophthalmol 1981; 65: 410-3

2 Shields MB. Primary angle-closure glaucoma. textbook of glaucoma Baltimore: Williams and Wilkins 1987: 16585

3 Spaeth GL. The normal development of the human anterior chamber angle: a new system of descriptive grading. Trans Ophthalmol Soc UK 1971; 91: 709-39.

4 Curran EJ. A new operation for glaucoma involving a new principle in the aetiology and treatment of chronic primary plaucoma. Arch Ophthalmol 1920; 49: 131-40.

5 Lowe RF. Primary creeping angle-closure glaucoma. $\mathrm{Br} F$ Ophthalmol 1964; 48: 544-50.

6 Mapstone R. Narrow angle glaucoma. Doc Ophthalmol Proc Ser 1980; 22: 185-93.

7 Spaeth GL. Ophthalmic surgery: principles and practice. Philadelphia: Saunders, 1982: chapter 10: 301-60.

8 Mapstone R. Angle-closure mechanisms in glaucoma. Sem Ophthalmol 1986; 1: 35-40.

9 Playfair TJ, Watson PG. Management of acute primary angleclosure glaucoma: a long-term follow-up of the results of peripheral iridectomy used as an initial procedure. $\operatorname{Br} \mathcal{F}$ Ophthalmol 1979; 63: 17-22.

10 Krupin T, Mitchell KB, Johnson MF, Becker B. The long-term effects of iridectomy for primary angle-closure glaucoma. Am f Ophthalmol 1979; 85: 506-9.

11 Floman N, Berson D, Landau L. Peripheral iridectomy in closed angle glaucoma - late complications. Brf Ophthalmol 1977; 61:101-4

12 Hyams SW, Friedman Z, Keroub C. Fellow eye in angleclosure glaucoma. Br $\mathcal{F}$ Ophthalmol 1974; 59: 207-10.

13 Wishart PK, Hitchings RA. Nd: YAG and dye laser iridotomy - a comparative study. Trans Ophthalmol Soc UK 1986; 105: $521-8$

4 Quigley HA. Long-term follow-up of laser iridotomy. Ophthalmology 1981; 88: 218-24.

15 Saunders DC. Acute closed-angle glaucoma and Nd-YAG laser iridotomy. Br $\mathcal{7}$ Ophthalmol 1990; 74: 523-5.

16 Gray RH, Hoare Nairne $\}$, Ayliffe WHR. Efficacy of Nd-YAG laser iridotomies in acute angle closure glaucoma. $\mathrm{Br} f$ Ophthalmol 1989; 73: 182-5.

17 Edwards RS. Behaviour of the fellow eye in acute angle-closure glaucoma. Br f Ophthalmol 1982; 66: 576-9.

18 Lowe RF. Acute angle-closure glaucoma. The second eye: an analysis of 200 cases. Br f Ophthalmol 1962; 46: 641-50.

19 Mapstone R. Mechanisms in ocular hypertension. $\mathrm{Br} f$ Ophthalomol 1979; 63: 325-30.

20 Mapstone $\mathrm{R}$. Acute shallowing of the anterior chamber. $\mathrm{Br} \mathcal{F}$ Ophthalmol 1981; 65: 446-51.

21 David R, Tessler Z, Yassur Y. Long-term outcome of primary acute angle-closure glaucoma. $\mathrm{Br} \mathcal{F}$ Ophthalmol 1985; 69: 261-2.

22 Wise JB. Iris sphincterotomy, iridotomy, and synechiotomy by linear incision with the argon laser. Ophthalmology 1985; 92: 641-5.

23 Schwartz LW, Moster MR, Spaeth GL, Wilson RP, Poryzees E. Nd: YAG laser iridotomy in glaucoma associated with closed or occludable angles. Am $\mathcal{F}$ Ophthalmol 1986; 102: $41-4$.

24 Pollack IP, Robin AL. A comparison of Nd: YAG and argon laser iridotomy. Ophthalmology 1984; 91: 1011-6. 